\title{
A Simulation Model of Rosa hybrida Growth Response to Constant Irradiance and Day and Night Temperatures
}

\author{
Douglas A. Hopper ${ }^{1}$ \\ Department of Horticulture, Colorado State University, Fort Collins, CO 80523 \\ P. Allen Hammer ${ }^{2}$ \\ Department of Horticulture, Purdue University, West Lafayette, IN 47907 \\ James R. Wilson ${ }^{3}$ \\ Department of Industrial Engineering, North Carolina State University, NC 27695-7906
}

Additional index words. canonical analysis, central composite design, light, irradiance, temperature

\begin{abstract}
This paper details the development and verification of ROSESIM, a computer simulation model of the growth of 'Royalty' roses (Rosa hybrida L.) based on experimentally observed growth responses from pinch until flowering under 15 combinations of constant photosynthetic photon flux (PPF), day temperature (DT), and night temperature (NT). Selected according to a rotatable central composite design, these treatment combinations represent commercial greenhouse conditions during the winter and spring in the midwestern United States; each selected condition was maintained in an environmental growth chamber having 12-hour photoperiods. ROSESIM incorporates regression models of four flower development characteristics (days from pinch to visible bud, first color, sepal reflex, and flowering) that are full quadratic polynomials in PPF, DT, and NT. ROSESIM also incorporates mathematical models of nine plant growth characteristics (stem length and the following fresh and dry weights: stem, leaf, flower, and total) based on data recorded every 10 days and at flowering. At each design point, a cubic regression in time (days from pinch) estimated the plant growth characteristics on intermediate days; then difference equations were developed to predict the resulting daily growth increments as third-degree polynomial functions of days from pinch, PPF, DT, and NT. ROSESIM was verified by plotting against time each simulated plant growth characteristic and the associated experimental observations for the eight factorial design points defining the region of interest. Moreover, one-way analysis of variance procedures were applied to the differences between ROSESIM predictions and the corresponding observed means for all 15 treatment combinations. At 20 days from pinch, significant differences $(P<0.05)$ were observed for all nine plant growth characteristics. At 30 and 40 days from pinch, only flower fresh and dry weights yielded significant differences; at flowering, none of the 13 selected responses yielded significant differences. These graphical and statistical comparisons provide good evidence of ROSESIM's ability to predict the growth response of 'Royalty' roses over a wide range of constant environmental conditions.
\end{abstract}

Functions relating plant growth characteristics to several environmental factors have successfully predicted plant response over set time intervals (Hammer and Langhans, 1976) or at flowering (Hopper and Hammer, 1991; Karlsson and Heins, 1986). Another approach to plant growth modeling consisted of developing linear or quadratic polynomials of time to depict responses over time of relative growth rate, net assimilation rate, and leaf area ratio for various cultivars (Fernandez and Miller, 1987) or to illustrate changes in stem length (SL) for cultivars grown under various environments (Hammer and Hopper, 1989). One study dynamically simulated plant growth in response to the complex energy balance of the greenhouse environment (Takakura et al., 1971). However, considerably more attention was directed toward thermal environmental relationships than to representing plant growth accurately. A dynamic representation of plant growth response to several environmental factors over time is desirable for predicting

Received for publication 28 Sept. 1992. Accepted for publication 12 Mar. 1994. Journal paper no. 13002 of the Purdue Univ. Agricultural Experiment Station. We thank E. G. Hill Co. for supplying the plants for this study. We gratefully acknowledge grants from the American Floral Endowment to support the PhD research of D.A.H. The cost of publishing this paper was defrayed in part by the payment of page charges. Under postal regulations, this paper therefore must be hereby marked advertisement solely to indicate this fact.

${ }^{1}$ Assistant professor.

${ }^{2}$ Professor.

${ }^{3}$ Professor changing plant response to constantly changing greenhouse conditions. By comparing growth observed at a time early in development with model predictions based on the measured environmental conditions, and then by predicting growth from that time until flower in response to expected environmental conditions, such a model could anticipate early in development how flowering shoot size and quality would be affected at flowering.

Many studies have illustrated the importance of photosynthetic photon flux (PPF) for promoting rose growth and flowering, whether it is received naturally (Mattson and Widmer, 1971) or applied supplementally (Armitage and Tsujita, 1979; Carpenter and Anderson, 1972; Cockshull, 1975; Moe, 1972; White and Richter, 1973). Net photosynthetic rate curves and the PPF saturation point have been determined for young leaves (Aiken and Hanan, 1975). PPF response functions have been determined to predict net photosynthesis for leaves 10 to 40 days old from 10 to 37C (Lieth and Pasian, 1990; Pasian and Lieth, 1989). Understanding these environmental effects on basic plant photosynthesis and partitioning to vegetative and reproductive tissues allows one to predict rose growth more accurately than by predicting photosynthesis alone.

More empirical approaches to plant growth modeling have used response surface techniques based on central composite experimental designs. Armitage et al. (1981) quantified flowering time response and general plant morphology of Tagetes patula L. to PPF, day temperature (DT), and night temperature (NT). Karlsson 
and Heins (1986) similarly predicted flowering, shoot length, and flower area response for Dendranthema grandiflora Tzvelev to the same three factors. Hammer and Langhans (1976) represented significant changes in fresh and dry weights of Helianthus annuus L. to daylength, light intensity, root temperature, DT, and NT. Kraszewski and Ormrod (1986) represented number and size of flowers, fresh weights, and dry weights of three plant species response to production and postharvest PPF. Yandell et al. (1988) constructed contour maps of tuber weight for two Solanum tuberosum L. cultivars in relation to $\mathrm{PPF}$, temperature, and $\mathrm{CO}_{2}$. Our study used such response surface techniques along with the dynamic nature of a simulation computer language to predict plant growth and development over daily time steps in response to constant PPF, DT, and NT. This paper describes the use of a new set of nine difference equations arranged in a 1-day time-step simulation model structure to predict growth increments in SL and fresh and dry weights of stem (FWS, DWS), leaf (FWL, DWL), and flower bud (FWF, DWF); the model also uses three developmental equations previously reported by Hopper and Hammer (1991) to determine plant development stages and to determine when to terminate the simulation at flowering.

\section{Materials and Methods}

Rose plant propagation and growth chamber conditions used to collect data on which this model is based were described previously (Hopper and Hammer, 1991). Thirty-two XXX (highest grade) 'Royalty' rose stock plants were obtained (E.G. Hill Co., Richmond, Ind.) and planted in 30-cm-diameter (15-liter) round plastic pots. The medium mix was 1 soil : 2 sphagnum peat : 2 perlite (by volume), $\mathrm{pH}$ 6.2, amended with the following nutrients (all per cubic meter): $744 \mathrm{~g}$ treble superphosphate, $496 \mathrm{~g}$ potassium nitrate, $496 \mathrm{~g}$ magnesium sulfate, $4 \mathrm{~kg}$ agricultural limestone, and $62 \mathrm{~g}$ fritted trace element mix (Robert B. Peters Co., Allentown, $\mathrm{Pa}$.). Plants were grown in a glasshouse with ambient solar irradiance and 22/16C day/night cycle. Liquid fertilization was applied with each irrigation (Hopper and Hammer, 1991). Plants used in treatments were started as single-node cuttings from these stock plants. Cuttings were placed in 6 sphagnum peat : 4 perlite : 1 vermiculite (by volume) medium, $\mathrm{pH} 5.5$, filling $6.5 \times 9.0 \times 6.0$ cm single-cell packs of 24 per $27 \times 54 \times 6.0$-cm flat (one cutting per cell). Cutting size varied in stem diameter $(0.5$ to $1.2 \mathrm{~cm}), \mathrm{SL}$ $(3.0$ to $10.0 \mathrm{~cm})$, and in leaf blade length $(8.0$ to $16.0 \mathrm{~cm})$ according to inherent variation in cutting material available for propagation. The basal end of the cutting was dipped in a commercial, powdered preparation (HormoRoot 2; Hortus Products, Newfoundland, N.J.) of $2 \%$ indole-3-butyric acid (IBA) before being placed in the medium.

The cuttings were held under intermittent mist (4 sec every 4 min during daylight) in a glass greenhouse for 4 weeks at constant $21 \mathrm{C}$ and ambient solar irradiance. During the study, a commercial antifungal material of 1-bromo-3-chloro-5,5-dimethyl-2,4imidazolidinedione (Agribrom; Great Lakes Chemical Corp., West Lafayette, Ind.) was added to the mist system via an injector (Dosomatic; A.H. Hummert Seed Co., St. Louis); this did not seem to alter rooting but prevented algae accumulation and improved overall cleanliness. After 4 weeks, the rooted cuttings were transplanted into $10-\mathrm{cm}(640-\mathrm{ml})$ square plastic pots in a medium as was described for the stock plants, and mist was gradually reduced over 1 week before moving plants to a growth chamber for treatment. Plants were acclimated to growth chamber treatment conditions for 7 to 10 days before hard-pinching to leave two five-leaflet leaves on each new plant shoot (only one new shoot per plant was allowed to grow). At the time of pinch, the 50 most uniform plants were selected for treatment, tagged for sampling, and completely randomized within the chamber.

Daylength was $12 \mathrm{~h}$ for all treatments. Day encompassed 1130 to $2330 \mathrm{HR}$ for chamber 1 or 1200 to $2400 \mathrm{HR}$ for chamber 2; this enabled convenient monitoring of day and night regimes. The 7- to 10-day period before pinch and start of the treatment allowed diurnal rhythms to adjust to the artificial daylength. Air temperatures were monitored and used for control using an aluminum-foilshielded probe suspended in the middle of the growth chamber; no leaf temperatures were recorded. Fertilizer was applied in daily irrigations with an automatic time-clock-regulated system. Two time clocks ( $24 \mathrm{~h}$ and $30 \mathrm{~min}$ ) wired in series allowed 5 to $10 \mathrm{~min}$ of irrigation for $15 \mathrm{~min}$ each day, depending on plant age and water requirements. The clocks engaged a submersible pump that supplied nutrient solution from a 114-liter plastic reservoir through a Chapin spaghetti-tube nonrecirculating irrigation system with two lead-weighted tubes in each pot. Each liter of nutrient solution supplied $250 \mathrm{mg} \cdot$ liter $^{-1}$ each of $\mathrm{N}$ and $\mathrm{K}$, while other essential elements were also provided according to the following formulation: $2.45 \mathrm{~g} \mathrm{KNO}_{3}, 1.74 \mathrm{~g} \mathrm{NH}_{4} \mathrm{NO}_{3}, 0.02 \mathrm{~g} \mathrm{MgSO}_{4}, 0.02 \mathrm{~g}$ soluble trace element mix (Robert B. Peters Co.), and $18.9 \mathrm{ml}$ of $75 \%$ technical grade $\mathrm{H}_{3} \mathrm{PO}_{4}$. Every 10 to 14 days, additional iron was supplied in the chelated form-monosodium ferric diethylenetriamine pentaacetate (sequestrene 330) — as a media drench of 5.8 g.liter ${ }^{-1}$.

Every 10 days starting from the day of pinch, growth from the topmost node was measured by destructively sampling 10 plants until day 40 and at flowering. Additional shoots below the topmost node were removed 10 days after pinch. Plant characteristics measured included number of nodes (NN), stem diameter, SL, and fresh and dry weights of stem, leaves, and flower bud. Flower development was measured for each plant by recording days from pinch until visible bud, days to first bud color, day to when sepals were reflexed $90^{\circ}$ from the main stem, and days until flowering, which was defined as the day when the first petal unfolded from the flower bud. Table 1 presents treatment combinations of PPF, DT, and NT that were selected according to the central composite rotatable experimental design. The order in which treatments were conducted was assigned at random; treatments were not blocked.

Model development. Using the SLAM II language (Pritsker, 1986) (Pritsker Corp., West Lafayette) permitted daily dynamic simulation of growth. A time increment of 1 day was selected since data are readily available from weather stations for daily integrated irradiance, whereas average DT and NT are recorded readily in production greenhouses. Shorter time steps, such as minute-byminute updates in a real-time simulation, did not agree with the overall objective and scale of the model to describe whole-plant growth from pinch until flowering. To devise a model to predict growth and development over a complete cut-flower crop cycle, plant data were destructively collected every 10 days. Real-time simulation would have required us to measure growth at much closer intervals than we used, while to construct this simulation model, we assumed that short-term changes could be represented by the integrated daily PPF and average daily temperatures.

The continuous model structure combined FORTRAN 77 and SLAM II code. This model structure required that difference equations depicting daily growth increments be developed by regression analysis of the plant characteristic response to the three environmental factors over time. To maintain homogeneity of variance, data were transformed by natural logarithm for SL, FWS, FWL, FWF, total bloom fresh weight (FWT), DWS, DWL, DWF, and total bloom dry weight (DWT). Characteristics related to time 
of flower bud development did not require any transformation. Since data were collected every 10 days, a regression analysis was necessary to estimate the daily growth increments for each treatment. The transformed data were regressed to estimate daily increases using SAS's PROC GLM procedure (SAS Institute, Cary, N.C.) by fitting cubic polynomial smoothing functions to each of the 15 unique treatment combinations and four additional replications $(n=5)$ of the design center point, having the following form:

$$
y_{t}=a_{0}+a_{1} t+a_{2} t^{2}+a_{3} t^{3} \text { for } t=11,12,13, \ldots
$$

where $y_{t}$ is the logarithm of the total for some predicted growth characteristic at time $t$ expressed in days from pinch. Natural-logtransformed data for each treatment were fit successively to linear, quadratic, and cubic polynomial functions of time; successively higher-order terms were included as long as adding the last term to the polynomial increased $R^{2}$ by $>1 \%$ and the $s_{b}$ value for the last term added was significant at $P=0.05$.

Each day the total growth for each treatment was calculated by Eq. [1], and the growth increment for that characteristic was calculated by $\Delta z_{t}=\exp \left[y_{t}\right]-\exp \left[y_{(t-1)}\right]$. SAS's GLM procedure fit an overall multiple regression model to the daily growth increments to obtain a generalized difference equation for each of the plant characteristics. This equation could predict a daily growth increment in response to the environment over the range of treatments in the design space and for the entire time from pinch until flowering. The polynomial form is a modification of a Taylor series algorithm for integrating differential equations, where the underlying unknown function may be linear or nonlinear (Conte and de Boor, 1980). Each difference equation includes 21 terms as follows (Table 2):

$$
\begin{aligned}
& \Delta z_{t}=b_{0}+b_{t} X_{t}+b_{t t} X_{t}^{2} \\
& +b_{1} X_{\mathrm{PPF}}+b_{2} X_{\mathrm{DT}}+b_{3} X_{\mathrm{NT}} \\
& +b_{11} X_{\mathrm{PPF}}^{2}+b_{22} X_{\mathrm{DT}}^{2}+b_{33} X_{\mathrm{NT}}^{2} \\
& +b_{12} X_{\mathrm{PPF}} X_{\mathrm{DT}}+b_{13} X_{\mathrm{PPF}} X_{\mathrm{NT}}+b_{23} X_{\mathrm{DT}} X_{\mathrm{NT}} \\
& +b_{t 1} X_{t} X_{\mathrm{PPF}}+b_{t 2} X_{t} X_{\mathrm{DT}}+b_{t 3} X_{t} X_{\mathrm{NT}} \\
& +b_{t 11} X_{t} X_{\mathrm{PPF}}^{2}+b_{t 22} X_{t} X_{\mathrm{DT}}^{2}+b_{t 33} X_{t} X_{\mathrm{NT}}^{2}+ \\
& b_{t 12} X_{t} X_{\mathrm{PPF}} X_{\mathrm{DT}}+b_{t 13} X_{t} X_{\mathrm{PPF}} X_{\mathrm{NT}}+b_{t 23} X_{t} X_{\mathrm{DT}} X_{\mathrm{NT}} \\
& \text { for } t=11,12,13, \ldots
\end{aligned}
$$

The terms $X_{\mathrm{PPF}}, X_{\mathrm{DT}}$, and $X_{\mathrm{NT}}$ are coded values of PPF, DT, and NT, respectively. Time was transformed to $X_{t}$ by dividing days from pinch by 10 ; using coded-factor and transformed-time terms restricted coefficient estimates of higher-order interactions from becoming excessively small, since SAS's PROC RSREG was

\begin{tabular}{|c|c|c|c|c|c|c|}
\hline \multirow{3}{*}{$\begin{array}{l}\text { Coded } \\
\text { value }\end{array}$} & \multicolumn{3}{|c|}{ Environmental factor } & & & \\
\hline & \multirow{2}{*}{$\begin{array}{c}\mathrm{PPF}^{\mathrm{Z}} \\
\left(\mu \mathrm{mol} \cdot \mathrm{m}^{-2} \cdot \mathrm{s}^{-1}\right)\end{array}$} & \multicolumn{2}{|c|}{ Temp $\left({ }^{\circ} \mathrm{C}\right)$} & & & \\
\hline & & $\overline{\text { Day }^{2}}$ & Night & & & \\
\hline-1.68 & 50 & 15 & 12 & & & \\
\hline-1.00 & 100 & 17 & 14 & & & \\
\hline 0.00 & 175 & 20 & 17 & & & \\
\hline 1.00 & 250 & 23 & 20 & & & \\
\hline \multirow[t]{3}{*}{1.68} & 300 & 25 & 22 & & & \\
\hline & \multicolumn{6}{|c|}{ Design matrix } \\
\hline & \multicolumn{3}{|c|}{ Coded axis ${ }^{\mathrm{y}}$} & \multicolumn{3}{|c|}{ Environmental setpoint } \\
\hline \multirow{2}{*}{$\begin{array}{l}\text { Treatment } \\
\text { no. }\end{array}$} & $X_{\mathrm{PPF}}$ & $X_{\mathrm{DT}}$ & $\overline{X_{\mathrm{NT}}}$ & \multirow{2}{*}{$\begin{array}{c}\text { PPF } \\
\left(\mu \mathrm{mol} \cdot \mathrm{m}^{-2} \cdot \mathrm{s}^{-1}\right)\end{array}$} & \multicolumn{2}{|c|}{ Temp $\left({ }^{\circ} \mathrm{C}\right)$} \\
\hline & & & & & $\overline{\text { Day }}$ & Night \\
\hline$\overline{1}$ & -1.68 & 0 & 0 & 50 & 20 & 17 \\
\hline 2 & -1 & -1 & -1 & 100 & 17 & 14 \\
\hline 3 & -1 & -1 & 1 & 100 & 17 & 20 \\
\hline 4 & -1 & 1 & 1 & 100 & 23 & 14 \\
\hline 5 & -1 & 1 & 1 & 100 & 23 & 20 \\
\hline 6 & 0 & 0 & 0 & 175 & 20 & 17 \\
\hline 7 & 0 & 0 & -1.68 & 175 & 20 & 12 \\
\hline 8 & 0 & 0 & 1.68 & 175 & 20 & 22 \\
\hline 9 & 0 & 1.68 & 0 & 175 & 25 & 17 \\
\hline 10 & 0 & -1.68 & 0 & 175 & 15 & 17 \\
\hline 11 & 1 & -1 & 1 & 250 & 17 & 20 \\
\hline 12 & 1 & 1 & 1 & 250 & 23 & 20 \\
\hline 13 & 1 & -1 & -1 & 250 & 17 & 14 \\
\hline 14 & 1 & 1 & -1 & 250 & 23 & 14 \\
\hline 15 & 1.68 & 0 & 0 & 300 & 20 & 17 \\
\hline
\end{tabular}
restricted to printing values with only eight digits to the right of the decimal. The dynamic nature of the SLAM II model, ROSESIM, arises from the using these difference equations, which are integrated by Euler's method at each time step (1 day) to update the values for each plant characteristic (state variables). The general equation for Euler's method (Conte and de Boor, 1980) starting at day 10 from pinch is

Table 1. Selected levels of environmental factors and the setpoints established according to the design matrix for 'Royalty' roses.

${ }^{\mathrm{Z}} \mathrm{PPF}=$ photosynthetic photon flux. Daylength (light period) is $12 \mathrm{~h}$ of continuous irradiation at the PPF indicated. Multiplication by the factor 0.0432 will convert constant instantaneous PPF for $12 \mathrm{~h}$ to the equivalent integrated daily value (e.g., $\mu \mathrm{mol} \cdot \mathrm{m}^{-2} \cdot \mathrm{s}^{-1}$ for $12 \mathrm{~h}$ daily $=4.32 \mu \mathrm{mol} \cdot \mathrm{m}^{-2} \cdot \mathrm{day}^{-1}$ ). Day and night temperatures are averages over the 12 -h period.

${ }^{\mathrm{y}}$ Coded values from the design matrix for the central composite rotatable design. $\mathrm{DT}=$ day temperature, $\mathrm{NT}=$ night temperature. 


\begin{tabular}{|c|c|c|c|c|c|c|c|}
\hline \multirow[b]{2}{*}{ Coefficient } & \multirow[b]{2}{*}{ Factor $^{2}$} & \multicolumn{6}{|c|}{ Characteristic } \\
\hline & & Estimate & $s_{b}$ & Estimate & $s_{b}$ & Estimate & $s_{b}$ \\
\hline & & \multicolumn{2}{|c|}{ Stem length } & \multicolumn{2}{|c|}{ Total fresh $w t$} & \multicolumn{2}{|c|}{ Total dry wt } \\
\hline$b_{0}$ & 1 & 1.03 & 0.249 & 0.746 & 0.112 & 0.0612 & 0.0210 \\
\hline$b_{t}$ & $X_{t}$ & 0.818 & 0.137 & -0.336 & 0.0617 & 0.00379 & 0.0116 \\
\hline$b_{t t}$ & $X_{t 2}$ & -0.211 & 0.0198 & 0.0777 & 0.00892 & 0.00584 & 0.00167 \\
\hline$b_{1}$ & $X_{\mathrm{PPF}}$ & 0.875 & 0.116 & 0.0293 & 0.0521 & 0.00317 & 0.00975 \\
\hline$b_{2}$ & $X_{\mathrm{DT}}$ & 1.12 & 0.123 & 0.0202 & 0.0554 & 0.0208 & 0.0104 \\
\hline$b_{3}$ & $X_{\mathrm{NT}}$ & 0.670 & 0.119 & -0.0239 & 0.0535 & 0.0125 & 0.0100 \\
\hline$b_{11}$ & $X_{\mathrm{PPF}} \times X_{\mathrm{PPF}}$ & -0.360 & 0.110 & -0.109 & 0.0495 & -0.0319 & 0.00926 \\
\hline$b_{12}$ & $X_{\mathrm{PPF}} \times X_{\mathrm{DT}}$ & 0.157 & 0.139 & -0.0185 & 0.0625 & -0.0123 & 0.0117 \\
\hline$b_{22}$ & $X_{\mathrm{DT}} \times X_{\mathrm{DT}}$ & 0.104 & 0.109 & 0.0570 & 0.0489 & 0.00468 & 0.00916 \\
\hline$b_{13}$ & $X_{\mathrm{PPF}} \times X_{\mathrm{NT}}$ & -0.0125 & 0.134 & -0.0397 & 0.0604 & -0.00911 & 0.0113 \\
\hline$b_{23}$ & $X_{\mathrm{DT}} \times X_{\mathrm{NT}}$ & 0.0584 & 0.141 & 0.0865 & 0.0632 & 0.0136 & 0.0118 \\
\hline$b_{33}$ & $X_{\mathrm{NT}} \times X_{\mathrm{NT}}$ & 0.253 & 0.110 & 0.0522 & 0.0496 & 0.00931 & 0.00929 \\
\hline$b_{t 1}$ & $X_{t} \times X_{\mathrm{PPF}}$ & -0.290 & 0.0364 & 0.0641 & 0.0164 & 0.0173 & 0.00307 \\
\hline$b_{12}$ & $X_{t} \times X_{\mathrm{DT}}$ & 0.416 & 0.0409 & 0.0147 & 0.0184 & -0.00253 & 0.00344 \\
\hline$b_{t 3}$ & $X_{t} \times X_{\mathrm{NT}}$ & -0.251 & 0.0384 & 0.0282 & 0.0173 & -0.00141 & 0.00324 \\
\hline$b_{t 11}$ & $X_{t} \times X_{\mathrm{PPF}} \times X_{\mathrm{PPF}}$ & 0.122 & 0.0325 & 0.0526 & 0.0146 & 0.0140 & 0.00274 \\
\hline$b_{t 12}$ & $X_{t} \times X_{\mathrm{PPF}} \times X_{\mathrm{DT}}$ & -0.0704 & 0.0411 & 0.0107 & 0.0185 & 0.00712 & 0.00347 \\
\hline$b_{t 22}$ & $X_{t} \times X_{\mathrm{DT}} \times X_{\mathrm{DT}}$ & -0.0638 & 0.0327 & -0.0311 & 0.0147 & -0.00483 & 0.00276 \\
\hline$b_{t 13}$ & $X_{t} \times X_{\mathrm{PPF}} \times X_{\mathrm{NT}}$ & -0.0216 & 0.0382 & 0.0144 & 0.0172 & 0.00258 & 0.00322 \\
\hline$b_{t 23}$ & $X_{t} \times X_{\mathrm{DT}} \times X_{\mathrm{NT}}$ & -0.0576 & 0.0422 & -0.0366 & 0.0190 & -0.00484 & 0.00356 \\
\hline$b_{t 33}$ & $X_{t} \times X_{\mathrm{NT}} \times X_{\mathrm{NT}}$ & 0.0849 & 0.0330 & -0.00892 & 0.0149 & -0.00195 & 0.00278 \\
\hline Significance & --- & \multicolumn{2}{|c|}{$* * *$} & & & & \\
\hline \multirow[t]{2}{*}{$R^{2}$} & -- & & & & & & \\
\hline & & & & & & & \\
\hline$b_{0}$ & 1 & 0.263 & 0.0352 & 0.115 & 0.0550 & 0.389 & 0.0381 \\
\hline$b_{t}$ & $X_{t}$ & -0.0944 & 0.0194 & 0.161 & 0.0303 & -0.425 & 0.0210 \\
\hline$b_{t t}$ & $X_{t 2}$ & 0.0137 & 0.00280 & -0.0330 & 0.00437 & 0.101 & 0.00303 \\
\hline$b_{1}$ & $X_{\mathrm{PPF}}$ & 0.0719 & 0.0163 & 0.152 & 0.0255 & -0.237 & 0.0177 \\
\hline$b_{2}$ & $X_{\mathrm{DT}}$ & 0.0455 & 0.0174 & 0.157 & 0.0272 & -0.198 & 0.0189 \\
\hline$b_{3}$ & $X_{\mathrm{NT}}$ & 0.000618 & 0.0168 & 0.0983 & 0.0262 & -0.127 & 0.0182 \\
\hline$b_{11}$ & $X_{\mathrm{PPF}} \times X_{\mathrm{PPF}}$ & -0.0394 & 0.0155 & -0.0496 & 0.0243 & 0.0105 & 0.0168 \\
\hline$b_{12}$ & $X_{\mathrm{PPF}} \times X_{\mathrm{DT}}$ & 0.0175 & 0.0196 & -0.0167 & 0.0307 & -0.0332 & 0.0213 \\
\hline$b_{22}$ & $X_{\mathrm{DT}} \times X_{\mathrm{DT}}$ & 0.00899 & 0.0154 & -0.0174 & 0.0240 & 0.0493 & 0.0167 \\
\hline$b_{13}$ & $X_{\mathrm{PPF}} \times X_{\mathrm{NT}}$ & -0.00606 & 0.0190 & -0.0389 & 0.0296 & 0.00680 & 0.0205 \\
\hline$b_{23}$ & $X_{\mathrm{DT}} \times X_{\mathrm{NT}}$ & -0.000263 & 0.0198 & 0.0249 & 0.0310 & 0.0532 & 0.0215 \\
\hline$b_{33}$ & $X_{\mathrm{NT}} \times X_{\mathrm{NT}}$ & 0.0229 & 0.0156 & 0.0375 & 0.0243 & -0.0307 & 0.0169 \\
\hline$b_{t 1}$ & $X_{t} \times X_{\mathrm{PPF}}$ & -0.00842 & 0.00514 & -0.0275 & 0.00803 & 0.116 & 0.00557 \\
\hline$b_{t 2}$ & $X_{t} \times X_{\mathrm{DT}}$ & -0.0138 & 0.00577 & -0.0657 & 0.00902 & 0.102 & 0.00625 \\
\hline$b_{t 3}$ & $X_{t} \times X_{\mathrm{NT}}$ & 0.00890 & 0.00543 & -0.0379 & 0.00849 & 0.0690 & 0.00589 \\
\hline$b_{t 11}$ & $X_{t} \times X_{\mathrm{PPF}} \times X_{\mathrm{PPF}}$ & 0.0189 & 0.00459 & 0.0210 & 0.00718 & 0.00131 & 0.00497 \\
\hline$b_{t 12}$ & $X_{t} \times X_{\mathrm{PPF}} \times X_{\mathrm{DT}}$ & -0.00587 & 0.00581 & -0.000490 & 0.00908 & 0.0247 & 0.00630 \\
\hline$b_{122}$ & $X_{t} \times X_{\mathrm{DT}} \times X_{\mathrm{DT}}$ & -0.00720 & 0.00462 & -0.00116 & 0.00722 & -0.0174 & 0.00500 \\
\hline$b_{t 13}$ & $X_{t} \times X_{\mathrm{PPF}} \times X_{\mathrm{NT}}$ & -0.000293 & 0.00540 & 0.00998 & 0.00844 & 0.00643 & 0.00585 \\
\hline$b_{123}$ & $X_{t} \times X_{\mathrm{DT}} \times X_{\mathrm{NT}}$ & -0.00378 & 0.00597 & -0.0146 & 0.00932 & -0.0147 & 0.00646 \\
\hline$b_{t 33}$ & $X_{t} \times X_{\mathrm{NT}} \times X_{\mathrm{NT}}$ & -0.00584 & 0.00467 & -0.0127 & 0.00729 & 0.0177 & 0.00506 \\
\hline Significance & --- & & & & & & \\
\hline$R^{2}$ & -- & & & & & & \\
\hline & & & & & & & \\
\hline$b_{0}$ & 1 & 0.00872 & 0.00625 & -0.00282 & 0.0123 & 0.0296 & 0.00401 \\
\hline$b_{t}$ & $X_{t}$ & 0.0100 & 0.00344 & 0.0558 & 0.00675 & -0.0426 & 0.00221 \\
\hline$b_{t t}$ & $X_{t 2}$ & -0.000124 & 0.000497 & -0.00936 & 0.000975 & 0.0122 & 0.000319 \\
\hline$b_{1}$ & $X_{\mathrm{PPF}}$ & 0.00632 & 0.00290 & 0.0294 & 0.00570 & -0.0314 & 0.00186 \\
\hline$b_{2}$ & $X_{\mathrm{DT}}$ & 0.0135 & 0.00309 & 0.0369 & 0.00606 & -0.0259 & 0.00198 \\
\hline$b_{3}$ & $X_{\mathrm{NT}}$ & 0.00635 & 0.00298 & 0.0239 & 0.00585 & -0.0134 & 0.00191 \\
\hline$b_{11}$ & $X_{\mathrm{PPF}} \times X_{\mathrm{PPF}}$ & -0.0145 & 0.00276 & -0.0156 & 0.00541 & 0.000118 & 0.00177 \\
\hline$b_{12}$ & $X_{\mathrm{PPF}} \times X_{\mathrm{DT}}$ & 0.000239 & 0.00349 & -0.00395 & 0.00684 & -0.00829 & 0.00224 \\
\hline$b_{22}$ & $X_{\mathrm{DT}} \times X_{\mathrm{DT}}$ & 0.00225 & 0.00273 & -0.00697 & 0.00535 & 0.00715 & 0.00175 \\
\hline$b_{13}$ & $X_{\mathrm{PPF}} \times X_{\mathrm{NT}}$ & -0.00178 & 0.00337 & -0.00928 & 0.00661 & 0.00137 & 0.00216 \\
\hline$b_{23}$ & $X_{\mathrm{DT}} \times X_{\mathrm{NT}}$ & 0.00340 & 0.00353 & 0.00557 & 0.00692 & 0.00395 & 0.00226 \\
\hline$b_{33}$ & $X_{\mathrm{NT}} \times X_{\mathrm{NT}}$ & 0.00503 & 0.00277 & 0.00594 & 0.00543 & -0.00438 & 0.00178 \\
\hline$b_{t 1}$ & $X_{t} \times X_{\mathrm{PPF}}$ & 0.00318 & 0.000913 & -0.00255 & 0.00179 & 0.0159 & 0.000586 \\
\hline$b_{12}$ & $X_{t} \times X_{\mathrm{DT}}$ & -0.00365 & 0.00103 & -0.0144 & 0.00201 & 0.0139 & 0.000658 \\
\hline$b_{t 3}$ & $X_{t} \times X_{\mathrm{NT}}$ & -0.00173 & 0.000965 & -0.00933 & 0.00189 & 0.00796 & 0.000619 \\
\hline$b_{t 11}$ & $X_{t} \times X_{\mathrm{PPF}} \times X_{\mathrm{PPF}}$ & 0.00638 & 0.000816 & 0.00622 & 0.00160 & 0.000678 & 0.000524 \\
\hline$b_{t 12}$ & $X_{t} \times X_{\mathrm{PPF}} \times X_{\mathrm{DT}}$ & 0.000925 & 0.00103 & 0.000993 & 0.00203 & 0.00520 & 0.000663 \\
\hline$b_{t 22}$ & $X_{t} \times X_{\mathrm{DT}} \times X_{\mathrm{DT}}$ & -0.00195 & 0.000821 & 0.000464 & 0.00161 & -0.00253 & 0.000527 \\
\hline$b_{t 13}$ & $X_{t} \times X_{\mathrm{PPF}} \times X_{\mathrm{NT}}$ & 0.000147 & 0.000960 & 0.00208 & 0.00188 & 0.000662 & 0.000616 \\
\hline$b_{123}$ & $X_{t} \times X_{\mathrm{DT}} \times X_{\mathrm{NT}}$ & -0.00178 & 0.00106 & -0.00231 & 0.00208 & -0.000563 & 0.000680 \\
\hline$b_{t 33}$ & $X_{t} \times X_{\mathrm{NT}} \times X_{\mathrm{NT}}$ & -0.00136 & 0.000830 & -0.00235 & 0.00163 & 0.00265 & 0.000532 \\
\hline Significance & --- & & & & & & \\
\hline$R^{2}$ & -- & & & & & & \\
\hline
\end{tabular}




$$
z_{t}=z_{t-1}+h \cdot \Delta z_{t}, t=10,11,12, \ldots
$$

where $z_{t}$ is the integrated state of a plant characteristic at time $t, z_{t-1}$ is the integrated state of a plant characteristic at the previous time, $t$ $-1, h$ is the step size, which was set constant to 1 day in ROSESIM, and $\Delta z$ is the growth increment for a plant characteristic over the 1-day time step. A plant characteristic was one of the measured growth characteristics, such as NN, SL, or DWT.

Since the first data were recorded 10 days after a hard pinch (a hard pinch is analogous to a harvest), ROSESIM was structured to make the first accurate prediction of growth at 10 days using a full quadratic response surface fit by SAS's RSREG procedure. Coefficient estimates are displayed in Table 3. PPF, DT, and NT are assumed to be constant over the total time from pinch until flowering. Future validation procedures will use Eq. [4], below, to simulate random integrated daily PPF for crop cycles for the whole year. Full quadratic model (10-term) regression equations to predict development (time to visible bud, time to first bud color, time to sepal reflex, and time to flower) were fit by SAS's PROC GLM procedure as presented previously by Hopper and Hammer (1991). Simulation would end when the incremented time from pinch exceeded the predicted time to flower.

Predicted growth curves under constant conditions of PPF, DT, and NT over the entire development period were used to verify the accuracy for predicting observed growth as measured in growth chambers. One-way SAS MANOVA procedures compared the difference of ROSESIM predictions from the observed data means for all 15 treatments (Table 4 ).
Extending ROSESIM to handle variable PPF. One of the ultimate objectives in developing ROSESIM is to predict rose growth under conditions of daily PPF variation. To do this, daily integrated radiometric values in Langleys/day from the West Lafayette weather station from November 1985 to February 1987 were converted to $\mathrm{mol} \cdot \mathrm{m}^{-2} \cdot \mathrm{day}^{-1}$ using conversion factors presented by Thimijian and Heins (1983). SAS's NLIN procedure was used to fit a sinusoidal model of the form

$$
\mathrm{PPF}_{\mathrm{JDAY}}=c_{0}+c_{1} \cdot \cos \left(0.0172 \cdot \mathrm{JDAY}+c_{2}\right)+\varepsilon_{\mathrm{JDAY}}
$$

where the constant 0.0172 forces a period of 1 year $(2 \pi / 365)$, and the other variables are defined as follows:

JDAY $=$ day of the year $(1$ Jan. $=1 ; 31$ Dec. $=365)$,

$\mathrm{PPF}_{\mathrm{JDAY}}=$ daily integrated PPF on day JDAY in $\mathrm{mol} \cdot \mathrm{m}^{-2} \cdot \mathrm{day}^{-1}$, $c_{0}=$ mean daily PPF averaged over the year (estimated by $\hat{c}_{0}=$ $24.4 \mathrm{~mol} \cdot \mathrm{m}^{-2} \cdot$ day $^{-1}$ ),

$c_{1}=$ maximum change from mean PPF (estimated by $\hat{c}_{1}=16.6$ $\mathrm{mol} \cdot \mathrm{m}^{-2} \cdot \mathrm{day}^{-1}$ ),

$c_{2}=$ phase shift in the cyclical trend (estimated by $\hat{c}_{2}=3.32$ radians, or $\approx 193$ days), and

$\varepsilon_{\mathrm{JDAY}}=$ observation error on day JDAY.

The statistics $\hat{c}_{0}, \hat{c}_{1}$, and $\hat{c}_{2}$ are estimated values derived by regression analysis. The estimated residuals $\left\{\hat{\varepsilon}_{\mathrm{JDAY}}: \mathrm{JDAY}=1,2,3, \ldots\right\}$ were nearly normal with a mean of zero. Generally, a regression procedure assumes an error structure that is random, so that an error on 1 day of the year is independent of the error on the previous

Table 3. Terms in seven full quadratic models and model significance for response surfaces at 10 days from pinch of 'Royalty' roses. Coefficients for equations used in the ROSESIM simulation model. The model form is $y=d_{0}+d_{1} X_{\mathrm{PPF}}+d_{2} X_{\mathrm{DT}}+d_{3} X_{\mathrm{NT}}+d_{11} X_{\mathrm{PPF}}^{2}+d_{22} X_{\mathrm{DT}}^{2}+d_{33} X^{2}{ }_{\mathrm{NT}}+d_{12} X_{\mathrm{PPF}} X_{\mathrm{DT}}$ $+d_{13} X_{\mathrm{PPF}} X_{\mathrm{NT}}+d_{23} X_{\mathrm{DT}} X_{\mathrm{NT}}$

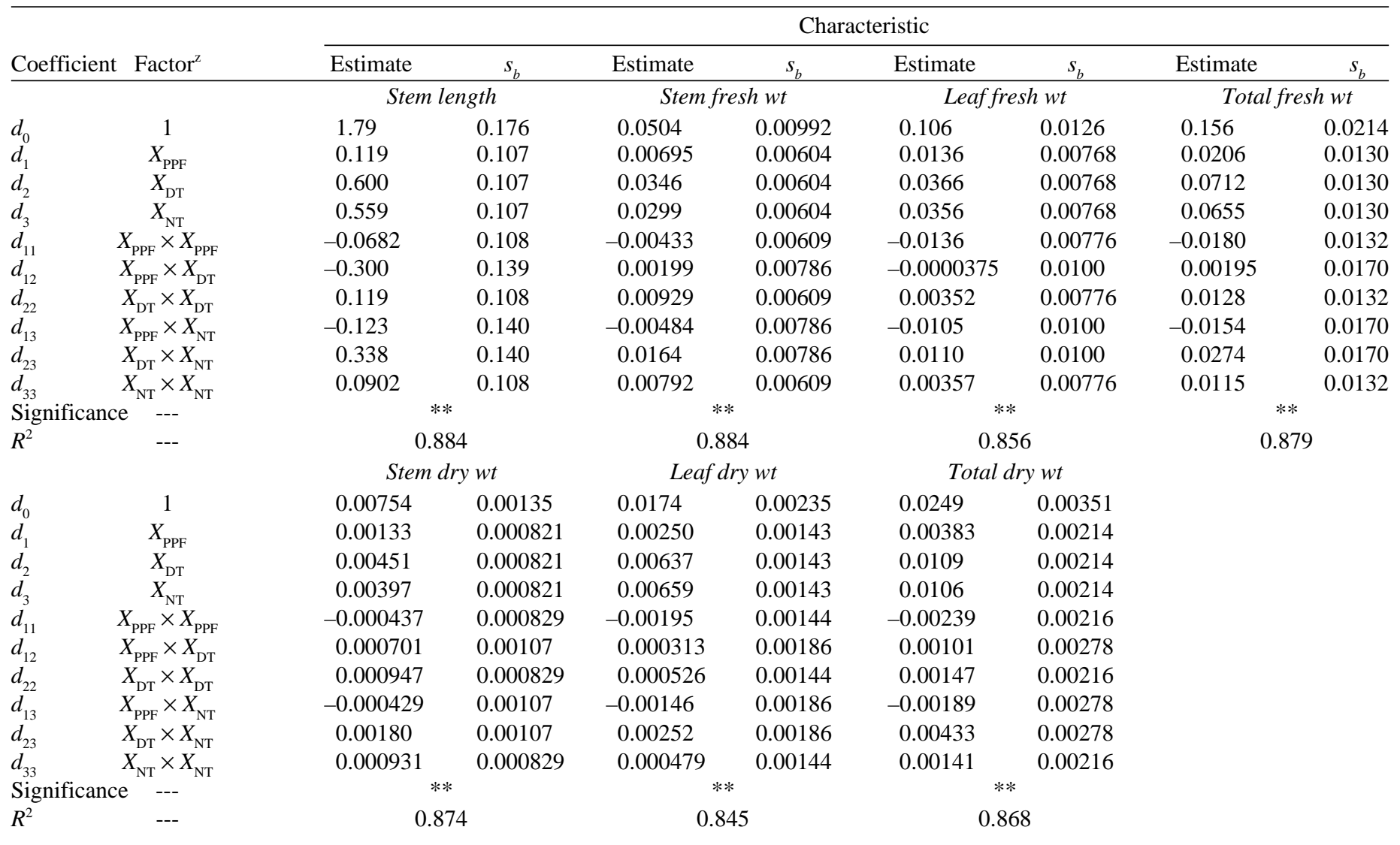

${ }^{\mathrm{z}}$ As shown in the design matrix in Table 1. $X_{\mathrm{PPF}}=$ coded photosynthetic photon flux; $X_{\mathrm{DT}}=$ coded day temperature; $X_{\mathrm{NT}}=$ coded night temperature. ${ }^{* *}$ Significant at $P=0.01$. 
Table 4. Significance of one-way analysis of variance (ANOVA) procedures comparing the difference of ROSESIM model predictions from the observed means of all 15 treatments at four times during 'Royalty' rose development. The error was estimated using the difference of model predictions from the five repetitions of the design center point.

\begin{tabular}{|c|c|c|c|c|}
\hline \multirow[b]{4}{*}{ Characteristic } & \multicolumn{4}{|c|}{ ANOVA significance $(P \text { value })^{z}$} \\
\hline & \multicolumn{4}{|c|}{ Time from pinch } \\
\hline & 20 & 30 & 40 & At \\
\hline & Days & Days & Days & flowering \\
\hline Stem length & 0.0250 & 0.548 & 0.777 & 0.0657 \\
\hline \multicolumn{5}{|l|}{ Fresh weight } \\
\hline Stem & 0.0037 & 0.619 & 0.864 & 0.366 \\
\hline Leaf & 0.0643 & 0.284 & 0.650 & 0.380 \\
\hline Flower & 0.0002 & 0.0001 & 0.0170 & 0.250 \\
\hline Total & 0.0019 & 0.376 & 0.564 & 0.243 \\
\hline \multicolumn{5}{|l|}{ Dry weight } \\
\hline Stem & 0.0151 & 0.618 & 0.939 & 0.653 \\
\hline Leaf & 0.0130 & 0.524 & 0.728 & 0.484 \\
\hline Flower & 0.0004 & 0.0001 & 0.0134 & 0.184 \\
\hline Total & 0.0004 & 0.557 & 0.783 & 0.452 \\
\hline Days to visible bud & --- & --- & --- & 0.955 \\
\hline Days to first color & --- & --- & --- & 0.456 \\
\hline Days to sepal reflex & --- & --- & --- & 0.420 \\
\hline Days to flower & --- & --- & --- & 0.913 \\
\hline
\end{tabular}

${ }^{\mathrm{z} P} \mathrm{v}$ value from SAS's PROC MANOVA. Values $>0.05$ indicate that ROSESIM predictions are not significantly different from the observed data means at $P=0.05$ when using the error estimate from the five repetitions of the design center point. Values $<0.05$ indicate that the model predictions were more distant from the data means of all 15 treatment combinations than the magnitude of variation observed between means for the five repetitions of the design center point.

day. However, analysis revealed a highly significant correlation between estimated residuals separated by a lag of 1 day. Consequently, we modeled the sequence of errors in Eq. [4] as a moving average process of order 1 (Box and Jenkins, 1976). Thus, in the extended version of ROSESIM, successive error terms were generated according to

$$
\varepsilon_{\mathrm{JDAY}}=u_{\mathrm{JDAY}}+\theta_{1} \cdot u_{\mathrm{JDAY}-1}, \text { for JDAY }=1,2,3, \ldots
$$

The estimated moving average parameter was $\hat{\theta}_{1} \approx 0.357$ and the shocks $\left\{u_{\mathrm{JDAY}}: \mathrm{JDAY}=1,2,3, \ldots\right\}$ driving the iterative scheme (Eq. [5]) were normal and independently distributed with a mean of zero and variance of $\hat{\sigma}_{u}^{2} \approx 77.1$. Equations [4] and [5] were fit to the data as presented in Fing 1.

To simulate $\mathrm{PPF}_{\mathrm{JDAY}}$ on a future day JDAY, we use the sampling variability of predictions based on the fitted Eq. [4]. The normal random variable $\mathrm{W}_{\text {JDAY }}$ is generated with

$$
\mathrm{E}\left[W_{\mathrm{JDAY}}\right]=\hat{c}_{0}+\hat{c}_{1} \cdot \cos \left(0.0172 \cdot \mathrm{JDAY}+\hat{c}_{2}\right)
$$

and

$$
\operatorname{Var}\left[W_{\mathrm{JDAY}}\right]=\hat{V}\left[\hat{c}_{0}+\hat{c}_{1} \cdot \cos \left(0.0172 \cdot \mathrm{JDAY}+\hat{c}_{2}\right)\right]
$$

where Eq. [7] is the NLIN-estimated variance of the statistic on the right-hand side of Eq. [6]; with the corresponding error, $\varepsilon_{\mathrm{JDAY}}$, generated according to Eq. [5].

The sampled value of $\mathrm{PPF}_{\mathrm{JDAY}}$ is

$$
\mathrm{PPF}_{\mathrm{JDAY}}=W_{\mathrm{JDAY}}+\varepsilon_{\mathrm{JDAY}}
$$

If the sampled value for PPF ${ }_{\text {JDAY }}$ for day JDAY falls outside of the design space as described in Table 1, then $\mathrm{PPF}_{\mathrm{JDAY}}$ is set to the value at the boundary of the design space.

SLAM II was selected to model this continuous development process because it is noted as a powerful and flexible simulation language with the ability to model a large variety of discrete and continuous processes while having many special features, such as queue processing and calls to random functions with many underlying distribution types (Pritsker, 1986). Randomness in predicted daily PPF was introduced through a call to RNORM, the generator for normally distributed random variables in SLAM II. If the

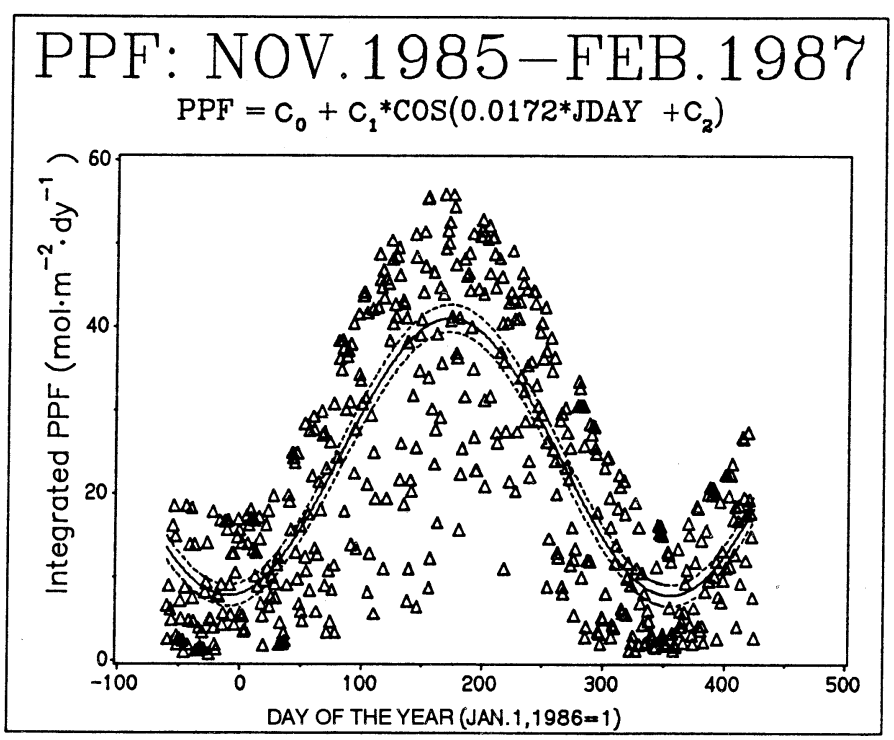

Fig. 1. Nonlinear regression fit of photosynthetic photon flux (PPF) to the day of the year. Period for data collection extends from 1 Nov. 1985 to 28 Feb. 1987, with 1 Jan. 1986 designated as day 1. The equation is used in the ROSESIM model to produce random daily PPF values for use in future validation procedures. 
random PPF exceeded the extremes in the original design, then the PPF value was set at the boundary of the design (Table 1). Since model predictions are based on an instantaneous PPF for $12 \mathrm{~h}$ each day, the random daily integrated estimate from Eq. [4] was converted to the 12-h continuous basis by multiplying by a factor of $23.1\left(\mathrm{~mol} \cdot \mathrm{m}^{-2} \cdot \mathrm{day}^{-1} \cdot 23.1=\mu \mathrm{mol} \cdot \mathrm{m}^{-2} \cdot \mathrm{s}^{-1}\right.$ continuously for $\left.12 \mathrm{~h}\right)$. The converted 12-h continuous value was used by the model to calculate predicted daily growth increments. Equation [4] and the conversion to a $12-\mathrm{h}$ continuous value have been added to the model structure to provide a mechanism for validation testing in future work with the model; random daily integrated PPF as would occur under actual greenhouse conditions could thus be simulated using the random nature of Eq. [4] to predict random daily integrated PPF for any crop period during a year.

\section{Results and Discussion}

Development rate varied greatly over the design space of the environmental factors studied. Earliest mean flowering occurred at 38 days, treatment 12 , with high PPF $\left(250 \mu \mathrm{mol} \cdot \mathrm{m}^{-2} \cdot \mathrm{s}^{-1}\right.$ or 10.8 $\mathrm{mol} \cdot \mathrm{m}^{-2} \cdot \mathrm{day}^{-1}$ ) and high day (23C) and night (20C) temperatures. Latest mean flowering occurred at 72 days, treatment 2, with low PPF $\left(100 \mu \mathrm{mol} \cdot \mathrm{m}^{-2} \cdot \mathrm{s}^{-1}\right.$ or $\left.4.32 \mathrm{~mol} \cdot \mathrm{m}^{-2} \cdot \mathrm{day}^{-1}\right)$ and low day $(17 \mathrm{C})$ and night (14C) temperatures. A wide range in time to flower between treatments suggested that it was necessary to include interaction terms of time with the coded environmental factors when developing multiple-regression difference equations for plant growth characteristics in ROSESIM. Since data were collected at intervals of at least 10 days, the data were smoothed to obtain daily incremental changes for each treatment. Fitting linear, quadratic, and cubic polynomial functions of time to natural-logtransformed data values of each treatment demonstrated that the cubic polynomial had improvements $>1 \%$ in $R^{2}$ over the quadratic form, and the $s_{b}$ value for the cubic term was significant at $P=0.05$. Predicted daily growth values from cubic polynomials, unique to each treatment run (19 total), were used to construct the 21-term difference equations for each of nine plant growth characteristics (Table 2).

The $R^{2}$ values for these equations varied from a low of 0.160 for FWS to a high of 0.915 for DWF. Such low values for several of the equations led to questioning whether the equations were adequate to model growth over the entire design space. However, when these 21-term difference equations were included, ROSESIM verified the agreement of model predictions with observed data for DWT, DWF, and SL when the environmental conditions were assumed constant over development (Figs. 2-4). These graphical representations of the predictions over the range of the original 15 treatment combinations (Table 1) show that the difference equations tracked growth closely even over the extreme responses to environments in the design space. This relationship also was tested statistically by comparing differences between ROSESIM predictions with the observed treatment means using one-way ANOVA at each 10 days of development after pinch and at flowering. ANOVA comparisons were not performed for 10 days from pinch since the response surface equations used to predict growth at 10 days were significant at $P=0.01$ (Table 3), so model predictions would be significantly close to treatment means by design. At 20 days after pinch, one-way ANOVA procedures (comparing differences between ROSESIM predictions with the observed treatment means) determined that model predictions for all characteristics except FWL were significantly different from some of the treatment means (Table 4). The significant differences between model predictions and actual means at 20 days indicate an inadequacy of the model to provide accurate predictions at that stage of development (Table 4). Fortunately, as development progressed to 30 and 40 days from pinch, differences for model predictions from the data means except for FWF and DWF were not significant. This verification procedure indicates that ROSESIM accurately represents growth responses at 30 and 40 days from pinch over the range of the design space; the magnitude of variation existing in the data is greater than the magnitude of the error that would be expressed as differences of model predictions from the treatment means. As time of development advanced to flowering, none of the predictions was significantly different from the data means at $P=0.05$, and only SL was significantly different at $P=0.10$. This result indicates that ROSESIM is also acceptable for predicting growth responses at flowering (or more precisely, that model predictions are not statistically different for $P=0.05$ from the measured treatment means at flowering). Since time to visible bud, first bud color, sepal reflex, and flower were each calculated by one response surface over the entire development time under constant conditions, the ANOVA test needed to be conducted only at flowering. Also, knowing that the response surface equations for all four of these flower development characteristics were significant, as presented by Hopper and Hammer (1991), the ANOVA test merely illustrated the significance of the fit by a different method.

The problem with producing accurate predictions at 20 days from pinch was evident for DWT (Fig. 2G) and SL (Fig. 4 A and $\mathrm{G})$. These errors appear as overpredictions due to inability of the single 21-term polynomial equation (which describes growth increments for any one growth characteristic over the very broad range of the design space) to describe a sigmoid curve shape fully when development was slow in response to low average daily temperatures (17/14C DT/NT). Any other equation form, such as the nonlinear Richards function, similarly cannot predict over the total development time for such a broad range of conditions with $<25$ estimated parameters. With the polynomial form we used, however, errors early in development ( $t \leq 20$ days) did not continue to be significant in later development $(t \geq 30$ days), since the magnitude of the errors early on was small (when daily incremental growth was small) compared to the magnitude of variation observed in later development (when the daily incremental growth was considerably larger). That is, daily growth increments increased dramatically between 20 and 30 days of development due to the exponential nature of plant growth. However, when the larger increments were added in later development, the size of these increments was visually large enough to make early errors appear insignificant, and statistically the errors at 20 days are less than the confidence interval at 30 days. It is mathematically interesting to note that this masking of early errors only occurs as growth is modeled by a polynomial summation over time (arithmetic fashion); using a Richards function or other exponential function would actually increase errors over time due to successive multiplications (the geometric nature of this approach was attempted and abandoned due to this inadequacy). The polynomial form in SLAM II structure is flexible enough to allow statistically acceptable prediction over 15 unique treatment combinations with a minimum of estimated parameters.

From our model, errors in prediction for DWF at 30 days are evident for treatments 12 and 15, both of which had rapid development and early flowering under high average daily temperatures of 23/20C DT/NT (Fig. $3 \mathrm{~F}$ and H). All of the plots for 30 and 40 days from pinch and at flowering indicated that ROSESIM predictions fall within the variability of the original data. Large gaps occur in recorded data for treatments 2 and 13 between 40 days and 


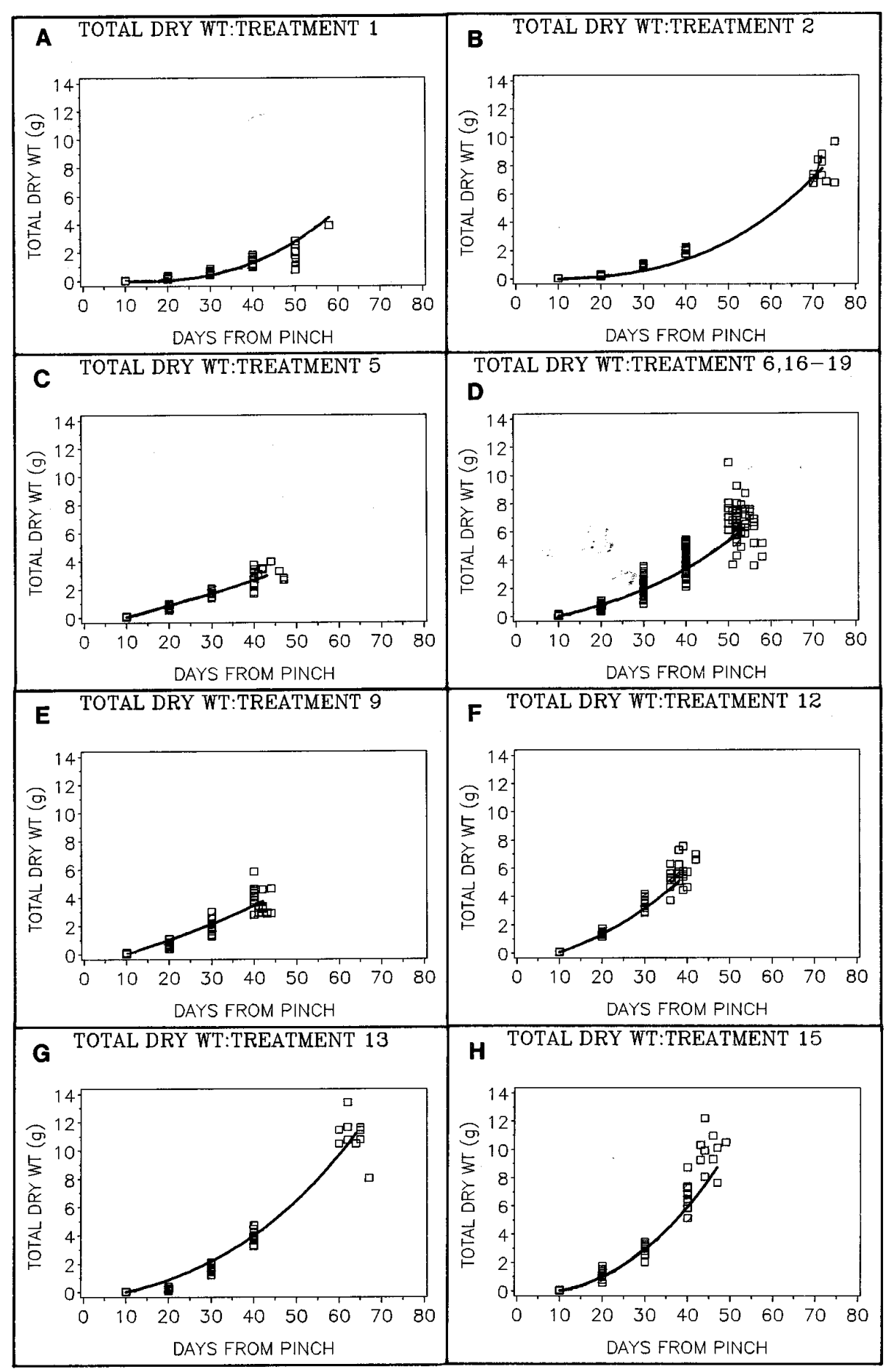

Fig. 2. Comparison of ROSESIM model predictions for 'Royalty' roses over time for total dry weights of eight treatments plotted with observed data. Simulations are based on equations fit to natural-logarithm-transformed data for smoothing with linear, quadratic, and cubic time terms. Antilog transforms were conducted for smoothed data for all treatments before fitting difference equations predicting daily growth increments.

\begin{tabular}{lcccc} 
Plot & Treatment & $\begin{array}{c}\mathrm{PPF}^{z} \\
\left(\mu \mathrm{mol} \cdot \mathrm{m}^{-2} \cdot \mathrm{s}^{-1}\right)\end{array}$ & $\begin{array}{c}\text { Day } \\
\text { temp } \\
\left({ }^{\circ} \mathrm{C}\right)\end{array}$ & $\begin{array}{c}\text { Night } \\
\text { temp } \\
\left({ }^{\circ} \mathrm{C}\right)\end{array}$ \\
\hline $\mathbf{A}$ & 1 & 50 & 20 & 17 \\
$\mathbf{B}$ & 2 & 100 & 17 & 14 \\
$\mathbf{C}$ & 5 & 100 & 23 & 20 \\
$\mathbf{D}$ & $6,16,17,18,19$ & 175 & 20 & 17 \\
$\mathbf{E}$ & 9 & 175 & 25 & 17 \\
$\mathbf{F}$ & 12 & 250 & 23 & 20 \\
$\mathbf{G}$ & 13 & 250 & 17 & 14 \\
$\mathbf{H}$ & 15 & 300 & 20 & 17 \\
\hline "PPF & & &
\end{tabular}

${ }^{2} \mathrm{PPF}=$ photosynthetic photon flux. 


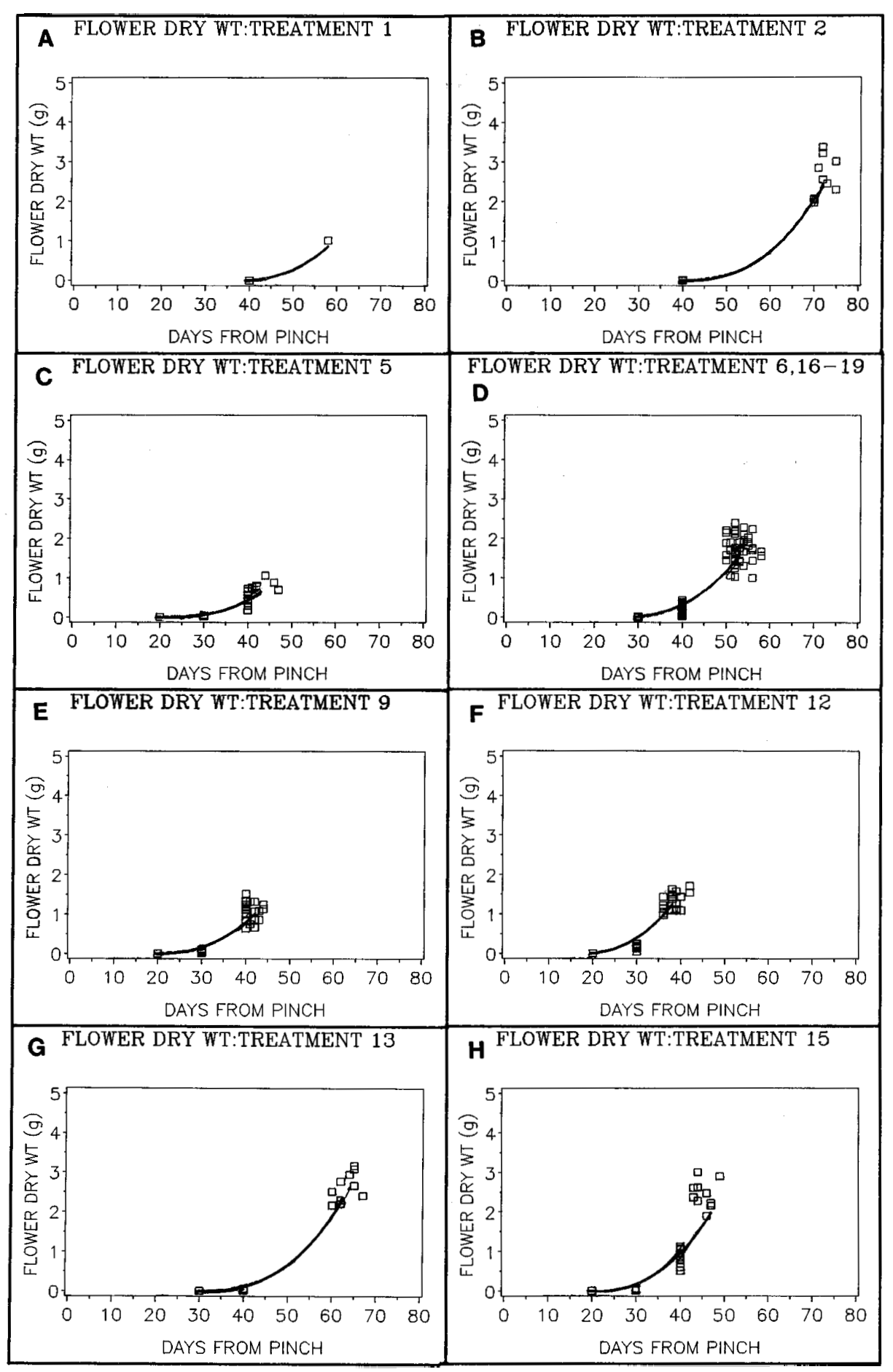

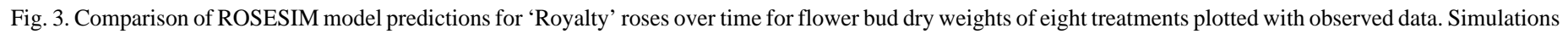

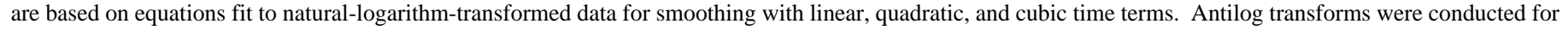
smoothed data for all treatments before fitting difference equations predicting daily growth increments.

\begin{tabular}{lcccc} 
Plot & Treatment & $\begin{array}{c}\text { DPF } \\
\mathrm{PPF}^{\mathrm{z}}\end{array}$ & $\begin{array}{c}\text { Day } \\
\text { temp } \\
\left(\mu \mathrm{mol} \cdot \mathrm{m}^{-2} \cdot \mathrm{s}^{-1}\right)\end{array}$ & $\begin{array}{c}\text { Night } \\
\text { temp } \\
\left({ }^{\circ} \mathrm{C}\right)\end{array}$ \\
\hline $\mathbf{A}$ & 1 & 50 & 20 & 17 \\
$\mathbf{B}$ & 2 & 100 & 17 & 14 \\
$\mathbf{C}$ & 5 & 100 & 23 & 20 \\
$\mathbf{D}$ & $6,16,17,18,19$ & 175 & 20 & 17 \\
$\mathbf{E}$ & 9 & 175 & 25 & 17 \\
$\mathbf{F}$ & 12 & 250 & 23 & 20 \\
$\mathbf{G}$ & 13 & 250 & 17 & 14 \\
$\mathbf{H}$ & 15 & 300 & 20 & 17 \\
\hline
\end{tabular}

${ }^{\mathrm{z}} \mathrm{PPF}=$ photosynthetic photon flux. 


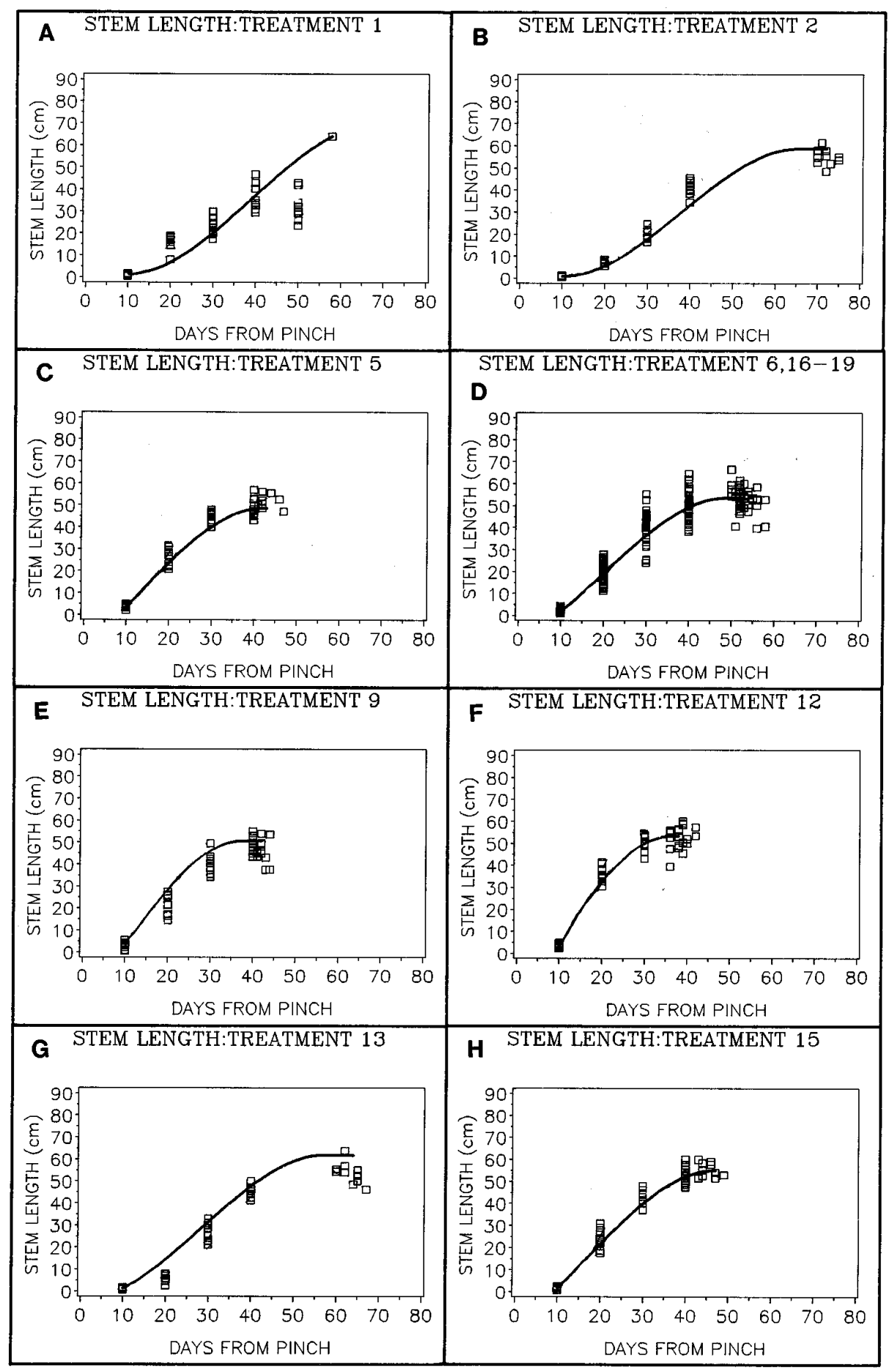

Fig. 4. Comparison of ROSESIM model predictions for 'Royalty' roses over time for stem lengths of eight treatments plotted with observed data. Simulations are based on equations fit to natural-logarithm-transformed data for smoothing with linear, quadratic, and cubic time terms. Antilog transforms were conducted for smoothed data for all treatments before fitting difference equations predicting daily growth increments.

\begin{tabular}{lcccc} 
Plot & Treatment & $\begin{array}{c}\mathrm{PPF}^{2} \\
\left(\mu \mathrm{mol} \cdot \mathrm{m}^{-2} \cdot \mathrm{s}^{-1}\right)\end{array}$ & $\begin{array}{c}\text { Day } \\
\text { temp } \\
\left({ }^{\circ} \mathrm{C}\right)\end{array}$ & $\begin{array}{c}\text { Night } \\
\text { temp } \\
\left({ }^{\circ} \mathrm{C}\right)\end{array}$ \\
\hline $\mathbf{A}$ & 1 & 50 & 20 & 17 \\
$\mathbf{B}$ & 2 & 100 & 17 & 14 \\
$\mathbf{C}$ & 5 & 100 & 23 & 20 \\
$\mathbf{D}$ & $6,16,17,18,19$ & 175 & 20 & 17 \\
$\mathbf{E}$ & 9 & 175 & 25 & 17 \\
$\mathbf{F}$ & 12 & 250 & 23 & 20 \\
$\mathbf{G}$ & 13 & 250 & 17 & 14 \\
$\mathbf{H}$ & 15 & 300 & 20 & 17 \\
\hline
\end{tabular}

${ }^{2} \mathrm{PPF}=$ photosynthetic photon flux. 
flowering (at $\approx 70$ days from pinch). The size of the growth chambers used limited the studies to 50 plants, and, with sampling every 10 days, there were not enough plants available to sample during that period, only at flowering. Further work at low temperatures should be conducted in larger chambers that would allow enough plants to be available to sample every 10 days until flowering. These plots and the ANOVA tests verify the accuracy of model prediction over time for a variety of treatments within the design space.

'Royalty' is a recently developed cultivar selected for its desirable commercial qualities. Our findings suggest 'Royalty' may exhibit longer SL than the older cultivars. Various cultivars were grown by Carpenter and Anderson (1972) in V-bottom ground benches in winter greenhouse conditions in Michigan, while our plants were grown in constant-environment growth chambers as described (Hopper and Hammer, 1991). These conditions, having set red to far-red ratios for PPF and set differences between day and night temperatures, also may have promoted the observed differences in SL. Carpenter and Anderson (1972) also found that 'Forever Yours', 'Shocking Pink', and 'Red American Beauty' irradiated for 20 or $21 \mathrm{~h}$ daily with fluorescent lamps (31.3 $\mathrm{W} / \mathrm{ft}^{2}$ or $470 \mu \mathrm{mol} \cdot \mathrm{m}^{-2} \cdot \mathrm{s}^{-1}$ ) had shorter SL and lower fresh weight compared with unlighted plants. Days until flower also were decreased significantly for 'Shocking Pink' and 'Red American Beauty' under supplemental irradiance. These results differ from those of our study. We found no significant differences in SL at the time of flowering, while increased PPF was associated with increased fresh and dry weights for all bloom parts. The conflict of our findings is most likely due to the longer daylength $(21 \mathrm{~h})$ used by Carpenter and Anderson than we used (12 h), which would result in a higher integrated daily PPF than we used in any treatment combination. Our results agree with respect to time of development since visible bud and flowering were observed under increased PPF earlier in our study. Possible explanations for these findings could be that plant surfaces may have been warmer with higher PPF, which in turn could have increased the rate of plant development, or that earlier floral initiation took place in response to higher PPF, as promoted by source-sink relationships.

Our results differ from those of Armitage and Tsujita (1979) using 'Caliente' roses. They reported shorter SLs under ambient irradiance $(30.4 \mathrm{~cm})$ and supplemental irradiance $(30.7 \mathrm{~cm})$ of 18 $\mathrm{h}$ at $110 \mu \mathrm{mol} \cdot \mathrm{m}^{-2} \cdot \mathrm{s}^{-1}$ for 0600 to $2400 \mathrm{HR}$ than we observed. Temperatures were constant at $21 \mathrm{C}$. The fresh weights for ambient (9.8 g) and supplemental PPF (11.0 g) conditions they reported also were less than those we observed or were predicted by ROSESIM for 20/17C DT/NT (data not shown). Growth under the lowest PPF of $50 \mu \mathrm{mol} \cdot \mathrm{m}^{-2} \cdot \mathrm{s}^{-1}$ more closely agreed with their results (fresh weight data not shown). Reduction in time to flower from 65.5 days under ambient irradiance to 51.4 days under supplemental irradiance agrees with results we observed and that were included in the response surface equations described previously (Hopper and Hammer, 1991).

Low temperatures have been shown to delay flower development (Hanan, 1979; Shanks et al., 1986; Zieslin et al., 1987). All of these findings are supported by our study when comparing treatments 2 and 5 (Fig. 2 B and C), both of which shared a 100 $\mu \mathrm{mol} \cdot \mathrm{m}^{-2} \cdot \mathrm{s}^{-1} \mathrm{PPF}$ but had different temperature regimes of $17 / 14 \mathrm{C}$ and 23/20C DT/NT, respectively. A similar comparison can be made with treatments 12 and 13 , both of which had a 250$\mu \mathrm{mol} \cdot \mathrm{m}^{-2} \cdot \mathrm{s}^{-1} \mathrm{PPF}$ but that were grown at $23 / 20 \mathrm{C}$ and $17 / 14 \mathrm{C} \mathrm{DT} /$ NT, respectively. Zieslin et al. (1987) recorded decreased yields due to flower bud abortion (blindness) at low temperature (14C), while Hanan (1979) observed increased flower bud malformation (bullheads) at low temperature $(<10 \mathrm{C})$. No such bud abortion was observed in our study due to low temperature, but flower buds from low-temperature treatments $(15 / 17 \mathrm{C})$ exhibited undesirable form when opening. Bud abortion was strongly promoted under very low PPF $\left(50 \mu \mathrm{mol} \cdot \mathrm{m}^{-2} \cdot \mathrm{s}^{-1}\right)$ in our study, with up to $90 \%$ of buds aborting. ROSESIM displays a warning message to the user if PPF values are $<50 \mu \mathrm{mol} \cdot \mathrm{m}^{-2} \cdot \mathrm{s}^{-1}$ for 10 days, but it does not attempt to predict abnormal development of any resulting blind shoots.

ROSESIM can predict rose shoot growth and daily development from pinch (or previous harvest) until flowering. Model predictions were accurate within the design space of 50 to 300 $\mu \mathrm{mol} \cdot \mathrm{m}^{-2} \cdot \mathrm{s}^{-1} \mathrm{PPF}, 15$ to $25 \mathrm{C} \mathrm{DT}$, and 12 to $22 \mathrm{C} \mathrm{NT}$. Caution must be used when attempting to predict growth in seasons or under constant conditions when either PPF or temperatures exceed the limits of the design space. For this reason, we recommend that this model be used for predicting rose growth only for constant conditions approximating the low-PPF months of October to March. Also, since 'Royalty' alone was used in this study, inferring the growth of other cultivars is not recommended. ROSESIM must be validated against roses grown under fluctuating conditions in a greenhouse before the model is used to alter commercial production practices. Should this validation be successful, ROSESIM would be a valuable tool for predicting 'Royalty' rose growth and development and adjusting greenhouse environmental control, especially if computer controls are used to manipulate greenhouse environmental conditions. The present model may be used with historical PPF values to study how rose growth would be expected to respond to varying daily natural solar radiation. Changes in the present FORTRAN code also could allow studies of rose response to fluctuating temperatures as one would observe under greenhouse conditions that did not have strict environmental control systems, but the model has not yet been validated under fluctuating conditions. ROSESIM also may be used in its present form to study growth under constant low or high temperature regimes while PPF is constant or with random daily PPF conditions. By selecting acceptable values for plant characteristics of primary interest, one could choose DT and NT setpoints that would produce the most desirable growth under anticipated low-PPF periods.

\section{Literature Cited}

Aiken, W.J. and J.J. Hanan. 1975. Photosynthesis in the rose; effect of light intensity, water potential and leaf age. J. Amer. Soc. Hort. Sci. 100:551-553.

Armitage, A.M. and M.J. Tsujita. 1979. The effect of nitrogen concentration and supplemental light on the growth and quality of Caliente roses. HortScience 14:614-615.

Armitage, A.M., W.H. Carlson, and C.E. Cress. 1981. Determination of flowering time and vegetative habit of Tagetes patula through response surface techniques. J. Amer. Soc. Hort. Sci. 106:632-638.

Box, G.E.P. and G.M. Jenkins. 1976. Time series analysis: Forecasting and control. revised ed. Holden-Day, San Francisco.

Carpenter, W.J. and G.A. Anderson. 1972. High intensity supplementary lighting increases yields of greenhouse roses. J. Amer. Soc. Hort. Sci. 97:331-334.

Cockshull, K.E. 1975. Roses II: The effects of supplementary light on winter bloom production. J. Hort. Sci. 50:193-206.

Conte, S. D., and C. de Boor. 1980. Elementary numerical analysis: An algorithmic approach. 3rd ed. McGrawHill, New York. p. 354-356, 359.

Fernandez, G.C.J. and J.C. Miller, Jr. 1987. Plant growth analysis of fieldgrown cowpeas J. Amer. Soc. Hort. Sci. 112:1044-1052.

Hammer, P.A. and R.W. Langhans. 1976. Growth models for Helianthus annuus L. and Zinnia elegans Jacq. J. Amer. Soc. Hort. Sci. 101:475479.

Hammer, P.A. and D.A. Hopper. 1989. Modeling stem elongation of Easter lilies grown under various production schemes. HortScience 
24:785-788.

Hanan, J.J. 1979. Observation of a low temperature effect on roses. J. Amer. Soc. Hort. Sci. 104:37-40.

Hopper, D.A. and P.A. Hammer. 1991. Regression models describing Rosa hybrida L. response to day/night temperature and photosynthetic photon flux. J. Amer. Soc. Hort. Sci. 116:609-617.

Karlsson, M.G. and R.D. Heins. 1986. Response surface analysis of flowering in chrysanthemum 'Bright Golden Anne'. J. Amer. Soc. Hort. Sci. 111:253-259.

Kraszewski, R.A. and D.P. Ormrod. 1986. Utilization of a response surface technique to study light acclimation of indoor flowering plants. J. Amer. Soc. Hort. Sci. 111:47-55.

Lieth, J.H. and C.C. Pasian. 1990. A model for net photosynthesis of rose leaves as a function of photosynthetically active radiation, leaf temperature, and leaf age. J. Amer. Soc. Hort Sci. 115:486-491.

Mattson, H.R. and R. E. Widmer. 1971. Effects of solar radiation, carbon dioxide, and soil fertilization on Rosa hybrida. J. Amer. Soc. Hort. Sci. 96:484-486.

Moe, R. 1972. Effect of day length, light intensity, and temperature on growth and flowering in roses. J. Amer. Soc. Hort. Sci. 97:796-800.

Pasian, C.C. and J.H. Lieth. 1989. Analysis of the response of net photosynthesis of rose leaves of varying ages to photosynthetically active radiation and temperature. J. Amer. Soc. Hort. Sci. 114:581-586. Pritsker, A.A.B. 1986. Introduction to simulation and SLAM II. 3rd ed. Wiley, New York.

Shanks, J.B., A.J. McArdle, G.D. Osnos, and H.G. Mityga. 1986. Greenhouse rose production with split night temperatures. J. Amer. Soc. Hort. Sci. 111:387-391.

Takakura, T., K.A. Jordan, and L.L. Boyd. 1971. Dynamic simulation of plant growth and environment in the greenhouse. Trans. Amer. Soc. Agr. Eng. 14:964-967.

Thimijian, R.W. and R.D. Heins. 1983. Photometric, radiometric, and quantum light units of measure: A review of procedures for interconversion. HortScience 18:818-822.

White, J.W. and D. Richter. 1973. Supplementary fluorescent lighting and low moisture stress improve growth of greenhouse roses. J. Amer. Soc. Hort. Sci. 98:605-607.

Yandell, B.S., A. Najar, R. Wheeler, and T.W. Tibbitts. 1988. Modeling the effects of light, carbon dioxide and temperature on the growth of potato. Crop Sci. 28:811-818.

Zieslin, N., E. Khayat, and Y. Mor. 1987. The response of rose plants to different night temperature regimes. J. Amer. Soc. Hort. Sci. 112:86-89. 